Okajimas Folia Anat. Jpn., 87(3): 123-128, November, 2010

\title{
Observing the bony canal structure of the human maxillary sinus in Japanese cadavers using cone beam CT
}

\author{
By \\ Iwao SATO ${ }^{1}$, Taisuke KAWAI ${ }^{2}$, Shunji YOSHIDA ${ }^{1}$, Yoko MIWA ${ }^{1}$, Kosuke IMURA ${ }^{1}$, \\ Rieko ASAUMI', Masataka SUNOHARA ${ }^{1}$ and Takashi YOSUE ${ }^{2}$ \\ ${ }^{1}$ Department of Anatomy \\ ${ }^{2}$ Department of Oral and Maxillofacial Radiology, School of Dentistry at Tokyo, Nippon Dental University, \\ 1-9-20 Fujimi Chiyoda-ku, Tokyo, 102-8159, Japan
}

- Received for Publication, September 13, 2010-

Key Words: bony structure, posterior superior alveolar artery, calcitonin gene-related peptide, maxillary sinus

\begin{abstract}
Summary: We observed the location of the posterior superior alveolar artery (PSAA) and nerve at the macroscopic level between the maxillary sinus (MS) and surrounding bone of the anterior region of the maxilla. This study was completed using cone beam computed tomography (CBCT) imaging of 19 human cadavers with 38 sides of Japanese origin (ranging in age from 59-94 years, mean $77.7 \pm 9.8$ years) that were prepared for this study. The bony canal structure of the inner surface of the maxilla was clearly apparent in our results, and the bony canals were classified into three types according to the structure along the course of the PSAA: canal-like, ditch-shaped tunnel and fragmented, and the lest sides were undefined.

Calcitonin gene-related peptide (CGRP)-positive fibers were identified along the PSAA in the bony canal of the maxilla by immunohistochemistry. The presence of the bony structure and CGRP-positive nerve fibers along the PSAA suggests that there is risk to the PSAA during surgery involving graft implant in the floor of the maxillary sinus.
\end{abstract}

\section{Introduction}

The maxillary blood supply forms a complex network on the surface of the maxilla. This vascular network is at risk during surgical treatment involving graft implant in the floor of the maxillary sinus. Many previous reports have described the distribution of the posterior superior alveolar artery (PSAA) of the maxillary sinus (MS) and its intraosseous or intrawall arteries. The maxillary vascular network of the lateral wall of the MS involves two arteries, the infraorbital artery and the PSAA, which are connected via anastomoses (Solar et al., 1999). These anastomoses contribute to the sinus membrane of the anterolateral wall of the sinus, and it is clinically important to know the location of the PSAA to avoid laceration during sinus floor elevation in the region of the molars. Solar et al. (1999) previously described the bony canal structure surrounding the PSAA. This bony canal structure is apparent in the MS in a large percentage of CT images (53\%) (Elian et al., 2005); 55\%, (Mardinger et al., 2007), CBCT images (71.4\%), (Ella et al., 2008) and cadaveric dissections (62.2\%) (Murakami et al.,
1994). The presence of the bony tunnel and nerve fibers running along the PSAA may pose a risk of vascular impairment or ischemia or of pain and inflammation during surgical treatments that involve drilling the alveolar bone. CBCT analysis of the morphological structure of the maxilla can provide useful information about the bony structure of the maxillary sinus.

The presence of CGRP-positive fibers along the PSAA indicates the capacity for vascular regulation, vasodilation and vasoconstriction (de Hoz et al., 2008). Furthermore, CGRP-immunoreactive fibers have been found in the nasal mucosa of patients with obstructive sleep apnea (Ju et al., 1991). The distribution of CGRPpositive fibers changes during vasodilatation and can be controlled by infusion of CGRP. The distribution of CGRP-positive fibers poses a risk during surgical treatment involving graft implant in the floor of the maxillary sinus. Thus, the distribution of CGRP-positive fibers along the PSAA in the lateral wall of the MS must be examined prior to treatment.

In the present study, we analyzed the bony structures within the maxillary vascular network of the lateral wall 
of the MS and the distribution of CGRP-positive fibers using immunohistochemistry and CBCT analyses.

\section{Materials and Methods}

Maxillae were examined in 19 human cadavers aged 59-94 years (mean, $77.7 \pm 9.8$ years; male, $80.0 \pm 10.7$ years; female, $75.1 \pm 8.5$ years) that were donated for human dissection. For each cadaver, both sides of the maxilla were studied. We eliminated four sides that had remarkable external injury and artifact with metal materials.

\section{Cone beam CT (CBCT) imaging}

We examined both sides of the maxilla in 19 human cadavers (34 sides; right sides 16; left sides, 18) using CBCT imaging (Alioth: ASAHI Roentgen Industry, Kyoto, Japan). Cone beam scans were carried out around the maxilla with a tube potential of $80 \mathrm{kV}$, a tube current of $4 \mathrm{~mA}$, and a cylindrical area of $51 \times 51 \mathrm{~mm}$ at high resolution (voxel size, $0.1 \mathrm{~mm}$ ). The Frankfurt horizontal $(\mathrm{FH})$ plane of the maxilla was placed parallel to the floor, and the region of interest (ROI) was set at the lateral region of the maxilla, placing the MS at the center of the ROI. Images were reconstructed from the CBCT data using proprietary Asahi Vision image reconstruction software (Asahi Roentgen Industry Co., Ltd).

\section{Immunohistochemistry}

After dissection, the MS was apparent in the maxilla. Samples underwent whole-mount immunohistochemical analysis of the MS and its blood vessels and nerves. The mucosa surrounding the MS was immersed in a 10 $\mathrm{mg} / \mathrm{L}$ solution of alizarin red $\mathrm{S}$ in ethanol to differentiate between peripheral nerves and vessels in 30 sides using a modification of previously reported methods (Yi et al., 2007). The solution was changed three times. Additional four sides were examined by whole-mount immunohistochemistry.

Whole-mount specimens were washed with distilled water for $24 \mathrm{hrs}$, incubated with $3 \% \mathrm{H}_{2} \mathrm{O}_{2}$ for $20 \mathrm{~min}$ to eliminate endogenous peroxidase activity and digested with $0.02 \%$ proteinase $\mathrm{K}$ (Wako, Tokyo, Japan) for 1 $\mathrm{hr}$ at $38^{\circ} \mathrm{C}$. After overnight fixation in $4 \%$ paraformaldehyde, the samples were washed with distilled water for $50 \mathrm{~min}$. They were then washed with phosphatebuffered saline (PBS) for $30 \mathrm{~min}$; sequentially incubated in $2.5 \%, 5 \%$ and $10 \%$ sucrose in PBS; and subjected to three freeze/thaw cycles. After overnight incubation with $2 \%$ Triton X-100 in PBS at $4{ }^{\circ} \mathrm{C}$, the samples were washed three times with PBS for $1 \mathrm{hr}$ and incubated for $1 \mathrm{hr}$ at room temperature with $2 \%$ normal goat serum in PBS (pH 7.2) containing $0.05 \%$ Tween 20 to prevent non-specific antibody binding. The samples were then incubated with rabbit polyclonal antibodies against
CGRP (1:1000; Biogenesis, NH, USA) or normal goat serum as a negative control. Subsequently, samples were washed three times with PBS for $1 \mathrm{hr}$ and incubated with HRP-conjugated goat anti-rabbit IgG according to the manufacturer's instructions (Santa Cruz Biotechnology, USA). The samples were then washed three times with PBS for $1 \mathrm{hr}$. Staining was visualized using $0.02 \% \mathrm{H}_{2} \mathrm{O}_{2}$ and $0.1 \%(1 \mathrm{mg} / \mathrm{ml})$ diaminobenzidine tetrahydrochloride in $0.1 \mathrm{M}$ Tris-HCl, $\mathrm{pH}$ 7.2. Images were acquired using a stereomicroscope (Leica MZ 16FA; Leica Microsystems, USA) with Leica Application Suite software (Leica Microsystems).

\section{Ethics}

Cadavers were obtained by consensual donation, according to the guidelines from the Law Concerning Body Donation for the Law Concerning Cadaver Dissection and Preservation (LCCDP).

\section{Results}

\section{Microscopic observations}

Two branches of the infraorbital artery, the middle superior alveolar artery and the anterior superior alveolar artery, were clearly identified on the lateral wall of the MS. The PSAA was identified parallel to the posterior superior alveolar nerve and branched several times around the lateral region of the MS. Numerous vessels and nerves forming complex fibers were observed near the floor region and the upper wall of the MS, and the PSAA and infraorbital artery formed anastomoses on the lateral wall of the MS (Figs. 1a-b). CGRP-positive nerves were clearly visible around the PSAA in the floor region of the MS. In addition, numerous anti-CGRP nerve fibers were scattered along the large vessels located on the lateral wall of the MS (Figs. 1c-d).

\section{CBCT analysis}

The bony canal structure of the inner surface of the maxilla was found and classified into three types: canallike, ditch-shaped tunnel, fragmented (Figs. $2 \mathrm{a}-\mathrm{c}$ and $3 \mathrm{a}-\mathrm{c})$. In 5 images ( 2 males and 3 females, representing $14.7 \%$ of the 34 images), the inner surface of the maxilla was canal-like structure along the course of the PSAA; in 23 images (11 males and 12 females, representing $67.6 \%$ of the 34 images), there was a ditch-shaped tunnel structure; and in 6 images ( 5 males and 1 female, representing $17.6 \%$ of the 34 images), there was a fragmented structure (Figs. 4a-c).

\section{Discussion}

Conventional dental imaging techniques such as dental and panoramic radiology provide limited informa- 


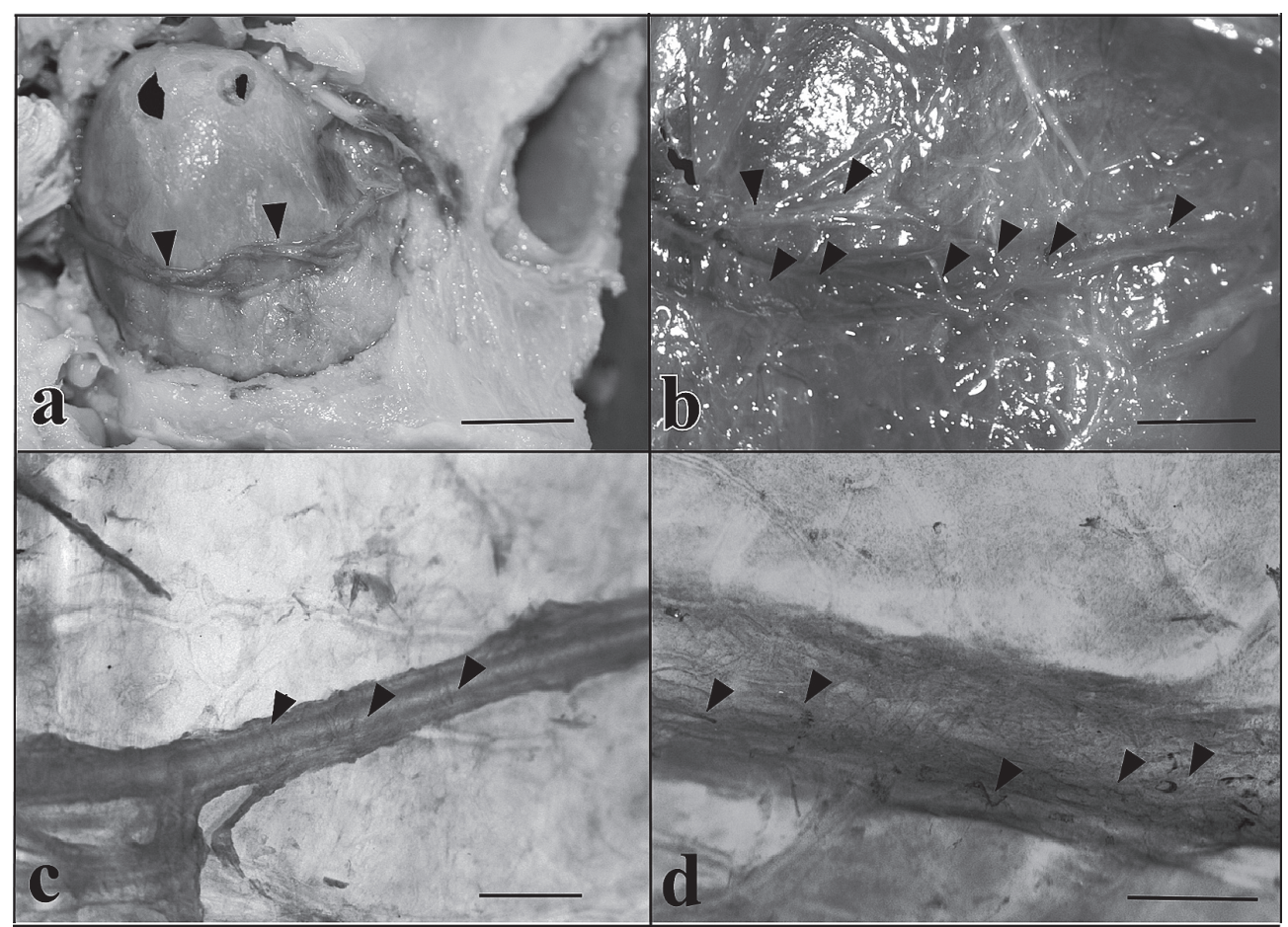

Fig. 1. (a) Lateral view of the maxillary sinus (MS) in the maxilla. The posterior superior alveolar artery (PSAA) (arrows) and the infraorbital artery (IOA) are shown in the lateral region of the MS by alizarin red S staining (black arrow heads ) $(\mathrm{bar}=1 \mathrm{~mm})$.

(b) Fine nervous fibers (black arrow heads) were identified along the PSAA (bar $=1 \mathrm{~mm}$ ).

(c) Distribution of CGRP-positive nerve fibers (black arrow heads ) identified by immunohistochemical staining at the macroscopic level (bar $=0.2 \mathrm{~mm}$ )

(d) Large magnification of Fig. 1c. CGRP expression (black arrow heads) was observed in small fibers along the PSAA (bar $=0.02 \mathrm{~mm})$.

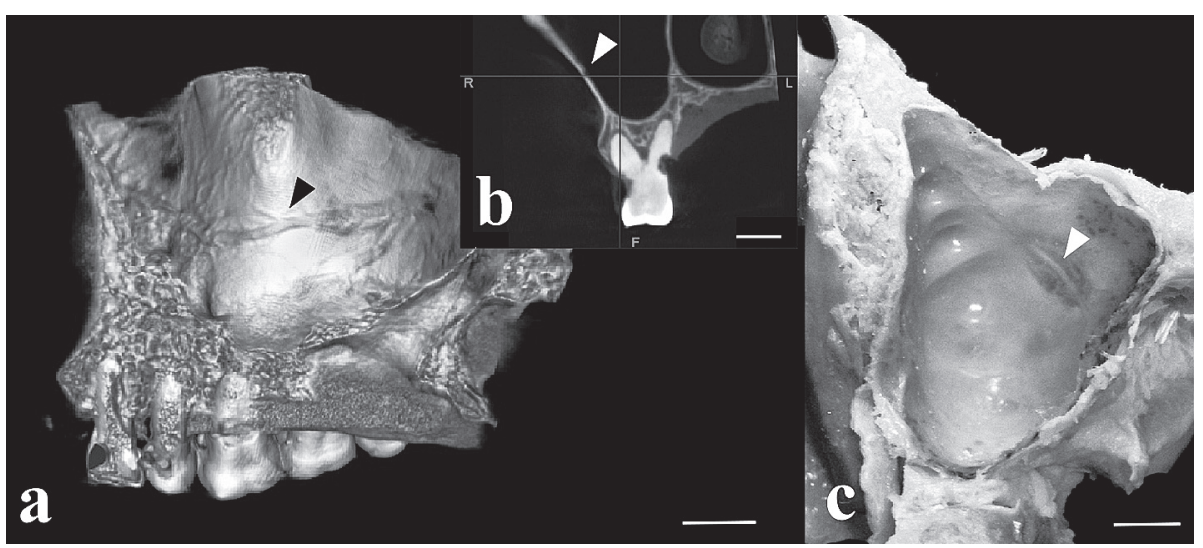

Fig. 2. (a) CBCT image showing a lateral view of the maxilla. A bone canal structure is shown with the PSAA (black arrowhead).

(b) CBCT (frontal) section image showing a bone canal structure (white arrowhead) on the buccal surface of the MS. The indicated location of white arrowhead is same region of black arrowhead in Fig. 2a.

(c) A view of the inner surface of the MS. The PSAA with nerve fibers were observed in the buccal surface region after the bony parts of the maxilla were removed. 

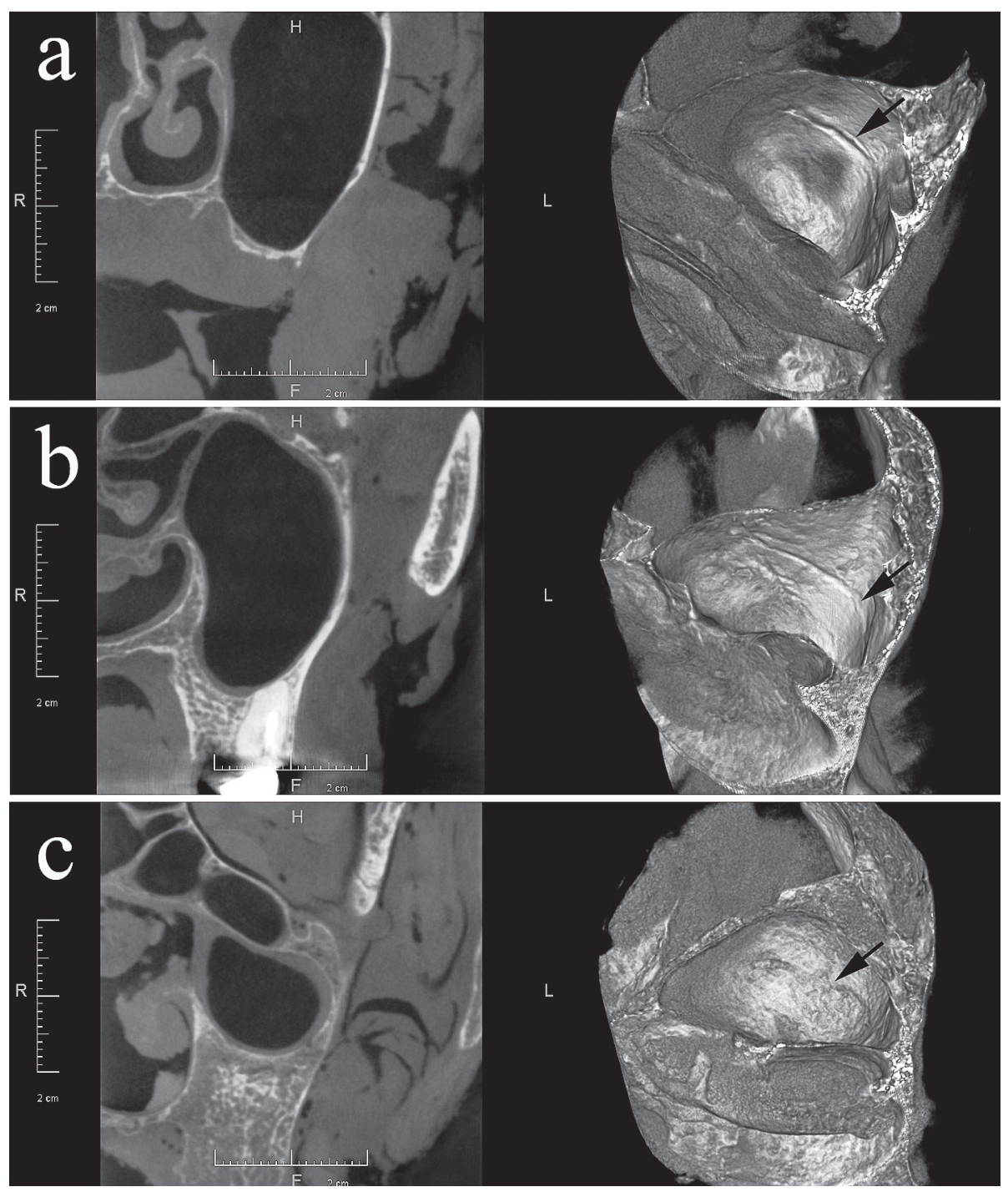

Fig. 3. Three patterns of CBCT image of the bony canals. Right side, 3D image; Left side, frontal section of CBCT image.

(a) CBCT image of a canal-like structure (black arrow) in a floor view of the MS in the maxilla.

(b) CBCT image of a ditch-shaped tunnel structure (black arrows) in a floor view of the MS in the maxilla.

(c) $\mathrm{CBCT}$ image of a fragmented structure (black arrows) in a floor view of the MS in the maxilla.

tion for visual examination because they produce only two-dimensional images of the mesiodistal and occlusalapical dimensions of the maxilla. CBCT systems have the ability to reconstruct full three-dimensional models of the maxilla, allowing precise definition of anatomical structures (Garg, 2007); (Monsour and Dudhia, 2008). Many reports have indicated that complex and anastomotic branches of the PSAA and the infraorbital artery are present in the lateral wall of the MS as determined by cadaver dissection (Murakami et al., 1994; Solar et al., 1999; Traxler et al., 1999; Rosano et al., 2009) and CT analysis (Mardinger et al., 2007; Ella et al., 2008). There are no available data, however, regard- ing visualization of the branches of the PSAA and the infraorbital artery using a combination of CBCT imaging and macroscopic analysis. Our macroscopic observations show that branches of the PSAA with CGRP-positive fibers were present in the maxillary sinuses of the Japanese cadavers. The varied appearance of the bony canal structure along the course of the PSAA has been reported in both CT (53\%, Elian et al., 2005; 55\%, Mardinger et al., 2007) and CBCT (71.4\%, Ella et al., 2008) studies. The CT system was used to generate overlapping $1.2-\mathrm{mm}$ axial cuts, and axial images were reconstructed into cross sections. In contrast, our CBCT system generated highresolution images (voxel size, $0.1 \mathrm{~mm}$ ). The smallest 

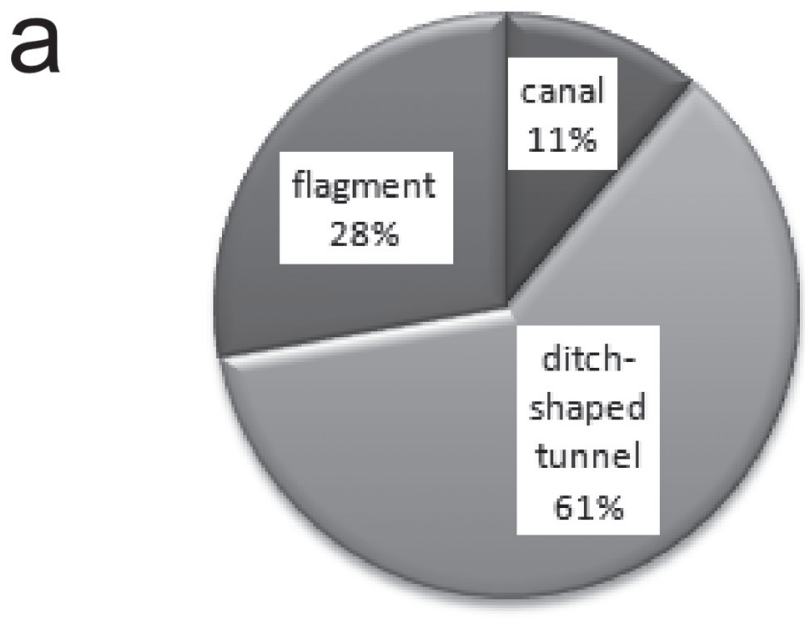

\section{b}

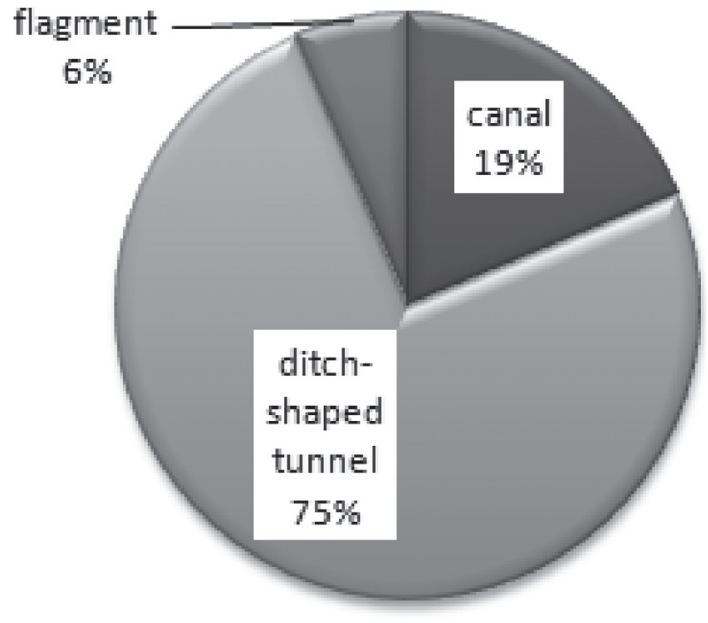

C

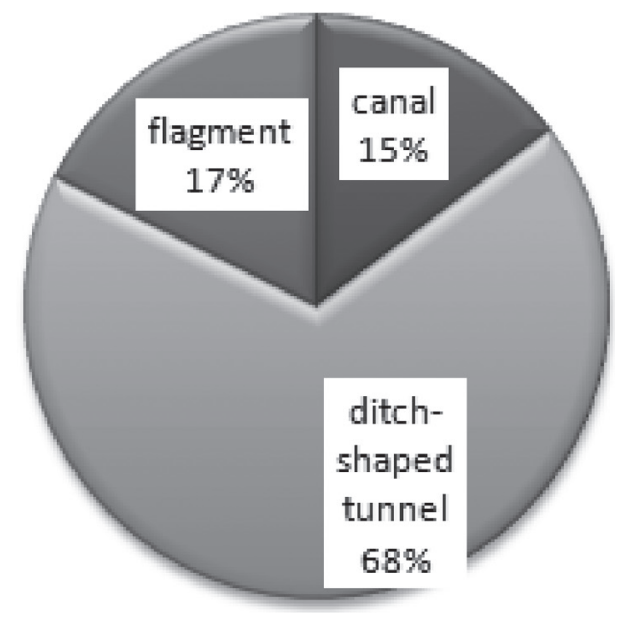

Fig. 4. Percentage of $\mathrm{CBCT}$ images showing the bony structure. Three types of structures were identified: canal-like, ditchshaped tunnel and fragmented. a, male; b, female; c, total. diameter of the PSAA has been reported to be less than $1 \mathrm{~mm}$ (Mardinger et al., 2007), and the largest diameter of the infraorbital artery has been reported to be $1.6 \mathrm{~mm}$ (Solar et al., 1999). Thus, most of the surrounding bony canal structure is not large enough to be detected by CT imaging. In the present study, this bony canal structure with canal-like and ditch-shaped tunnel were evident in $82.3 \%$ of the images, greater than the value of $71.4 \%$ observed in a previous CBCT analysis (Ella et al., 2008). Furthermore, we inspected the bony canal structure by maxillary dissection of our specimens following CBCT analysis. We also defined this structure in detail as one of three types: canal-like, ditch-shaped tunnel or fragmented. The ditch-shaped tunnel structure was the most common (67.6\% of the 34 images), and the thin wall of this structure may pose a risk during implant surgery. The bony canal structure may be formed by the inflammation of maxillary membrane with paranasal sinuses, which may be due to infection, allergy or autoimmune issues. Especially most of our examined specimens were mainly elderly subjects, and therefore formed canal structure may be concerned with aging.

CGRP is released by nociceptive sensory nerves. In addition, CGRP-positive fibers are associated with the regulation of blood vessels supplying local tendons and contribute to vasodilation (de Hoz et al., 2008; Danielson et al., 2006). Our results demonstrate that CGRP-positive fibers are located along the PSAA in the bony structure of the maxilla. It has been suggested that CGRP-positive fibers mediate blood vessel regulation. Dental surgeons must be mindful of this point when performing maxillary sinus graft procedures for dental implants. Post-operative pain and discomfort can occur in the maxillary sinus floor following surgical treatment with sinus floor elevation (Trombelli et al., 2010). Post-operative pain along with posterior superior alveolar nerve damage is related to anatomical variations in the paranasal sinuses (Whittet, 1992; Solar et al., 1999). In the present study, numerous nerve branches with CGRP-positive fibers were identified along the vessels in the human maxillary sinuses that we examined. This region is susceptible to inflammation following implant placement (Penarrocha-Diago et al., 2009). In conclusion, identification of the arterial supply and of the innervation of the maxillary sinus area is essential prior to dental surgical treatment, and CBCT imaging is required to observe the intraosseous vessels in this region.

\section{References}

1) Danielson $P$, Alfredson $H$ and Forsgren S. Distribution of general (PGP 9.5) and sensory (substance P/CGRP) innervations in the human patellar tendon. Knee Surg Sports Traumatol Arthrosc 2006; 14:125-132.

2) de Hoz R, Ramirez AI, Salazar JJ, Rojas B, Ramirez JM and Trivino A. Substance P and calcitonin gene-related peptide intrin- 
sic choroidal neurons in human choroidal whole-mounts. Histol Histopathol 2008; 23:1249-1258.

3) Elian N, Wallace S, Cho SC, Jalbout ZN and Froum S. Distribution of the maxillary artery as it relates to sinus floor augmentation. Int J Oral Maxillofac Implants 2005; 20:784-787.

4) Ella B, Sedarat $C$, Noble Rda $C$, Normand E, Lauverjat $Y$, Siberchicot F, Caix P and Zwetyenga N. Vascular connections of the lateral wall of the sinus: surgical effect in sinus augmentation. Int J Oral Maxillofac Implants 2008; 23:1047-1052.

5) Garg AK. Dental implant imaging: TeraRecon's Dental 3D Cone Beam Computed Tomography System. Dent Implantol Update 2007; 18:41-45.

6) Ju G, Liu S and Zang X. Peptidergic innervation of the mammalian anterior pituitary. NIPS 1991; 6:26-28.

7) Mardinger O, Abba M, Hirshberg A and Schwartz-Arad D. Prevalence, diameter and course of the maxillary intraosseous vascular canal with relation to sinus augmentation procedure: a radiographic study. Int J Oral Maxillofac Surg 2007; 36:735-738.

8) Monsour PA and Dudhia R. Implant radiography and radiology. Aust Dent J 2008; 53:S11-S25.

9) Murakami G, Ohtsuka K, Sato I, Moriyama H, Shimada K and Tomita H. The superior alveolar nerves: their topographical relationship and distribution to the maxillary sinus in human adults. Okajimas Folia Anat Jpn 1994; 70:319-328.
10) Penarrocha-Diago M, Boronat-Lopez A and Garcia-Mira B. Inflammatory implant periapical lesion: etiology, diagnosis, and treatment--presentation of 7 cases. J Oral Maxillofac Surg 2009; 67:168-173.

11) Rosano G, Taschieri S, Gaudy JF and Del Fabbro M. Maxillary sinus vascularization: a cadaveric study. J Craniofac Surg 2009; 20:940-943.

12) Solar P, Geyerhofer U, Traxler H, Windisch A, Ulm C and Watzek G. Blood supply to the maxillary sinus relevant to sinus floor elevation procedures. Clin Oral Implants Res 1999; 10:34-44.

13) Traxler H, Windisch A, Geyerhofer U, Surd R, Solar P and Firbas W. Arterial blood supply of the maxillary sinus. Clin Anat. 1999; 12:417-421.

14) Trombelli L, Minenna P, Franceschetti G, Minenna L and Farina R. Transcrestal sinus floor elevation with a minimally invasive technique. J Periodontol 2010; 81:158-166.

15) Whittet HB. Infraorbital nerve dehiscence: the anatomic cause of maxillary sinus "vacuum headache"? Otolaryngol Head Neck Surg 1992; 107:21-28.

16) Yi S-Q, Ohta T, Tsuchida A, Terayama H, Naito M, Li J, Wang $\mathrm{H}-\mathrm{X}$, Yi N, Tanaka S and Itoh M. Surgical anatomy of innervation of the gallbladder in humans and Suncus murinus with special reference to morphological understanding of gallstone formation after gastrectomy. World J Gastroenterol 2007; 13:2066-2071. 